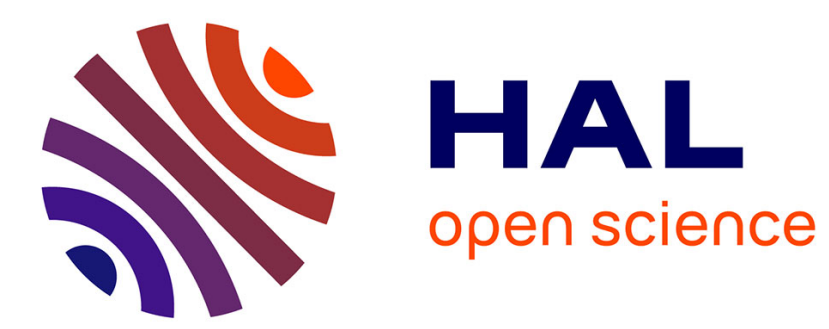

\title{
On the Mahler measure in several variables
}

Francesco Amoroso

\section{To cite this version:}

Francesco Amoroso. On the Mahler measure in several variables. Bulletin of the London Mathematical

Society, 2008, 40 (4), pp.619-630. hal-00424262

\section{HAL Id: hal-00424262 \\ https://hal.science/hal-00424262}

Submitted on 14 Oct 2009

HAL is a multi-disciplinary open access archive for the deposit and dissemination of scientific research documents, whether they are published or not. The documents may come from teaching and research institutions in France or abroad, or from public or private research centers.
L'archive ouverte pluridisciplinaire HAL, est destinée au dépôt et à la diffusion de documents scientifiques de niveau recherche, publiés ou non, émanant des établissements d'enseignement et de recherche français ou étrangers, des laboratoires publics ou privés. 


\title{
On the Mahler measure in several variables.*
}

\author{
Francesco Amoroso
}

\begin{abstract}
.
If the total degree of a polynomial in $n \geq 2$ variables of dimension $n \mathrm{~s}$ bounded by a double exponential function in $n$, we show that its Mahler measure is bounded from below by an absolute constant $>1$.
\end{abstract}

2000 Mathematics Subject Classification 11G50, 11J81, 14G40.

\section{Introduction.}

In early 1933 Lehmer (cf. [9], 13, p. 476) wrote

"The following problem arises immediately. If $\varepsilon$ is a positive quantity, to find a polynomial of the form $f(x)=x^{r}+a_{1} x^{r-1}+\ldots+a_{r}$ where the $a$ 's are integers, such that the absolute value of the product of those roots of $f$ which lie outside the unit circle, lies between 1 and $1+\varepsilon$. (...) Whether or not the problem has a solution for $\varepsilon<0.176$ we do not know."

Let $P(x)=a\left(x-\alpha_{1}\right) \cdots\left(x-\alpha_{d}\right)$ be a polynomial with complex coefficients. We define its Mahler measure as

$$
M(P)=|a| \prod_{j=1}^{d} \max \left\{1,\left|\alpha_{j}\right|\right\} .
$$

By Kronecker's theorem $M(f)=1$ for an irreducible polynomial $f \in \mathbb{Z}[x]$ if and only if $f \neq \pm x$ or if $\pm f$ is a cyclotomic polynomial. Lehmer's problem is equivalent to the following

Conjecture 1.1 Let $f \in \mathbb{Z}[x]$ be a nonconstant irreducible polynomial. Assume $f \neq \pm x$ and that $\pm f$ is not a cyclotomic polynomial. Then

$$
M(f) \geq C
$$

for some absolute constant $C>1$.

\footnotetext{
${ }^{*}$ The final version of this article has been published in the Bull. London. Math. Vol. 40, no. 4, published by the London Mathematical Society
} 
The best known result in the direction of this conjecture is Dobrowolski's lower bound

$$
M(f) \geq C\left(\frac{\log D}{\log \log D}\right)^{-3}
$$

which holds for any $f$ of degree $D \geq 2$ as in conjecture 1.1. Here $C$ is an absolute constant. In the original statement ([6]) $C=1 / 1200$; later Voutier ([14]) shows that one can take $C=1 / 4$.

In this paper we are interested in the generalization of conjecture 1.1 to polynomials in several variables. Let $n$ be a positive integer and $\mathbf{x}=\left(x_{1}, \ldots, x_{n}\right)$. We define the Mahler measure of a polynomial $P \in \mathbb{C}[\mathbf{x}]$ as

$$
M(P)=\exp \int_{0}^{1} \ldots \int_{0}^{1} \log \left|P\left(e^{2 \pi i t_{1}}, \ldots, e^{2 \pi i t_{n}}\right)\right| d t_{1} \ldots d t_{n} .
$$

We remark that by Jensen's formula this definition coincide with the previous one if $n=1$. Further, $M(f) \geq 1$ for $f \in \mathbb{Z}[\mathbf{x}]$, as is easily seen by induction on $n$. An analogous of Kronecker theorem is known. Following Schinzel, we say that an irreducible $f \in \mathbb{Z}[\mathbf{x}]$ is an extended cyclotomic polynomial if there exist a cyclotomic polynomial $\phi$ and $\boldsymbol{\lambda}, \boldsymbol{\mu} \in \mathbb{Z}^{n}$ such that

$$
f(\mathbf{x})= \pm \mathbf{x}^{\boldsymbol{\lambda}} \phi\left(\mathbf{x}^{\boldsymbol{\mu}}\right) .
$$

In other words, $f \in \mathbb{Z}[\mathbf{x}]$ is extended cyclotomic if and only if the hypersurface $\{f=0\}$ in $\mathbb{G}_{\mathrm{m}}^{n}$ is a torsion variety (i.e. an union of translates of subtori by torsion points) defined and irreducible over the rationals. Kronecker's theorem generalizes as follows. Let $f \in \mathbb{Z}[\mathbf{x}]$ be irreducible. Then $M(f)=1$ if and only if $f= \pm x_{j}$ or if $f$ is an extended cyclotomic polynomial ([3], [7] and [13] independently).

We remark that conjecture 1.1 implies the lower bound

$$
M(f) \geq C
$$

for any nonconstant irreducible $f \in \mathbb{Z}[\mathbf{x}]$ such that $f \neq \pm x_{j}$ and $f$ not extended cyclotomic. In this statement, $C$ is the same as in conjecture 1.1. This is an easy consequence of the following result of Lawton (see [8]). Let $P \in \mathbb{C}[\mathbf{x}]$, and define, for $\lambda \in \mathbb{N}^{n}$,

$$
q(\boldsymbol{\lambda})=\min \left\{\max \left|\mu_{j}\right| \mid \boldsymbol{\mu} \in \mathbb{Z}^{n} \backslash\{\mathbf{0}\}, \boldsymbol{\lambda} \cdot \boldsymbol{\mu}=0\right\} .
$$

and

$$
P_{\boldsymbol{\lambda}}(t)=P\left(t^{\lambda_{1}}, \ldots, t^{\lambda_{n}}\right) \in \mathbb{C}[t]
$$

Then,

$$
\lim _{q(\boldsymbol{\lambda}) \rightarrow+\infty} M\left(P_{\boldsymbol{\lambda}}\right)=M(P) .
$$

Unfortunately, the quoted result of Lawton cannot be used to deduce an analogue in several variables of Dobrowolski's result. Nevertheless, the method of Dobrowolski's proof has been generalized to several variables in [1]. Let

$$
f=\sum_{\boldsymbol{\lambda}} f_{\boldsymbol{\lambda}} \mathbf{x}^{\boldsymbol{\lambda}} \in \mathbb{Z}[\mathbf{x}]
$$


be irreducible. Following Smyth ([12]) we define the $\operatorname{dimension} \operatorname{dim} f$ as the dimension in $\mathbb{R}^{n}$ of the convex-hull of the set $\left\{\boldsymbol{\lambda}\right.$ such that $\left.f_{\boldsymbol{\lambda}} \neq 0\right\}$. It is easy to see that $\operatorname{dim} f$ is the smallest integer $m$ such that $f$ comes from a polynomial in $m$ variables by a monomial transformation:

$$
f(\mathbf{x})=\mathbf{x}^{\boldsymbol{\lambda}_{0}} g\left(\mathbf{x}^{\boldsymbol{\lambda}_{1}}, \ldots, \mathbf{x}^{\boldsymbol{\lambda}_{m}}\right)
$$

where $g \in \mathbf{x}\left[y_{1}, \ldots, y_{m}\right]$ and $\boldsymbol{\lambda}_{0}, \ldots, \boldsymbol{\lambda}_{m} \in \mathbb{Z}^{n}$. Note that this formula implies the equality $M(f)=M(g)$. Further, $\operatorname{dim} f$ is the codimension of the stabilizer of the hypersurface $\{f=0\}$ in $\mathbb{G}_{\mathrm{m}}^{n}$.

In [1] the authors show that for an irreducible polynomial $f \in \mathbb{Z}[\mathbf{x}]$ of dimension $n$, we have

$$
\log M(f) \geq \frac{1}{C(n+1)^{1+4 / n} n^{2}} \cdot \frac{(\log ((n+1) \log ((n+1) D)))^{2+1 / n}}{(\log ((n+1) D))^{1+2 / n}}
$$

where $C$ is a positive constant. Note that the exponents on the error terms are slightly better than the exponent 3 in Dobrowolski's result.

The above considerations suggest that in some sense a generalized Lehmer's conjecture could be easier than the original one. More precisely, for any $n \geq 2$ we propose the following weaker form of Lehmer's conjecture.

Conjecture 1.2 There exists an absolute constant $C>1$ such that for any irreducible $f \in \mathbb{Z}\left[x_{1}, \ldots, x_{n}\right]$ of dimension $n \geq 2$ we have

$$
M(f) \geq C .
$$

Our main result shows that any eventual counterexample to this conjecture must have a very high degree with respect to $n$.

Theorem 1.3 Let $f \in \mathbb{Z}\left[x_{1}, \ldots, x_{n}\right]$ be an irreducible polynomial of dimension $n$. Let $D$ be the maximum of its partial degrees. Assume $n \geq 9$ and

$$
D \leq 3^{2^{n}} .
$$

Then

$$
\log M(f) \geq \frac{1}{23} .
$$

Let $f \in \mathbb{Z}\left[x_{1}, \ldots, x_{n}\right]$ be an irreducible polynomial. The normalized height $\hat{h}(V)$ of the hypersurface $V=\{f=0\} \subset \mathbb{G}_{\mathrm{m}}^{n}$ is $\log M(f)$. If $V$ is not defined over the rationals, see $\S 2$ for the definition of $\hat{h}(V)$. Theorem 1.3 generalizes to a lower bound for the normalized height of a hypersurface defined and irreducible over a number field (theorem 3.4 and remark 3.6). Moreover, in theorem 3.5 we bound from below $\hat{h}(V)$ by a function $c(n, D)>0$ depending on $n$ and on $D=\operatorname{deg}(V)$. Unfortunately, $c(n, D)$ might go to zero according to the growth of $D$ with respect to $n$. 
We finally mention that one formulate an even more optimistic conjecture. In [4], Boyd asked whether the function

$$
m(n)=\inf \left\{M(f) \text { such that } f \in \mathbb{Z}\left[x_{1}, \ldots, x_{n}\right] \text { is irreducible and } \operatorname{dim} f=n\right\}
$$

tends to infinity with $n$. Concerning this problem, the best known sequence of polynomials is the simplest one: $f_{n}(x)=x_{1}+\cdots+x_{n}$. For these polynomials we have

$$
\log M\left(f_{n}\right) \sim \frac{1}{2} \log n
$$

(see [12], [10]).

Acknowledgements. I would like to express my gratitude to Martin Sombra and Evelina Viada for numerous helpful conversations on the subject of this paper. The term involving the Arithmetic Hilbert Function in proposition 3.2 comes from a suggestion of M. Sombra.

\section{Overview of the proof.}

Let $F$ be an auxiliary polynomial vanishing on a geometrically irreducible variety $V \subset \mathbb{G}_{\mathrm{m}}^{n}$. Then, if some inequalities concerning degrees and heights hold, $F$ must vanish on the translates of $V$ by $p$-torsion points, at least for small primes $p$. In [2] we exploit this vanishing principle to obtain a lower bound for the normalized height of $V$, under the assumption that $V$ is not a translate of a subtorus. The main new idea behind the proof of theorem 1.3 is the following: the above vanishing principle make use of the fact that $V$ is $p$-adically close to $\zeta V$ for all $p$-torsion points $\boldsymbol{\zeta}$. But all the translates of $V$ by $p$-torsion points are close to each other. Thus, we replace the vanishing principle used in [2] by a symmetric vanishing principle. For technical reasons, it is more convenient to use an interpolation determinant than an auxiliary function. This will be done in proposition 3.2, which contains all the information needed for the proof of theorem 1.3.

\section{Notation and preliminary results.}

\subsection{Normalized height, essential minimum.}

Let $K$ be a number field and let $V$ be a hypersurface in $\mathbb{G}_{\mathrm{m}}^{n}$ defined over $K$ :

$$
V=\left\{\boldsymbol{\alpha} \in \mathbb{G}_{\mathrm{m}}^{n} \text { such that } f(\boldsymbol{\alpha})=0\right\}
$$

with $f \in K[\mathbf{x}]$ square-free. Let $\mathcal{M}_{K}$ be the set of places of $K$. We define

$$
\hat{h}(V)=\frac{1}{[K: \mathbb{Q}]} \sum_{v \in \mathcal{M}_{K}}\left[K_{v}: \mathbb{Q}_{v}\right] \log M_{v}(f)
$$

where if $v$ is non archimedean $M_{v}(f)$ is the maximum of the $v$-adic absolute values of the coefficients of $f$ and if $v$ is an archimedean place associated with the embedding $\sigma: K \hookrightarrow \overline{\mathbb{Q}}$

$$
M_{v}(f)=M(\sigma f) .
$$


We recall that $\hat{h}$ is an additive function. Let $V$ be a reduced hypersurface, say $V=W_{1} \cup \cdots \cup W_{l}$ where $W_{1}, \ldots, W_{l}$ are geometrically irreducible hypersurfaces. Then $\hat{h}(V)=\hat{h}\left(W_{1}\right)+\cdots+\hat{h}\left(W_{l}\right)$. For a more general definition of $\hat{h}$ and for more of its properties, see [5].

Let $V$ be a $K$-irreducible hypersurface. For $\theta \geq 0$ we denote

$$
V(\theta)=\{\boldsymbol{\alpha} \in V(\overline{\mathbb{Q}}) \text { such that } \hat{h}(\boldsymbol{\alpha}) \leq \theta\},
$$

where $\hat{h}(\boldsymbol{\alpha})=h\left(\left(1: \alpha_{1}: \cdots, \alpha_{n}\right)\right)$ and $h$ is the Weil height on the projective space. Hence $V(0)$ is the set of torsion points on $V$. Let define the essential minimum $\hat{\mu}^{\text {ess }}(V)$ of $V$ as the infimum of the set of $\theta \geq 0$ such that $V(\theta)$ is Zariski dense in $V$. By a special case of Zhang's inequality (see [15]) we have

$$
\hat{\mu}^{\mathrm{ess}}(V) \leq \frac{\hat{h}(V)}{\operatorname{deg}(V)} \leq n \hat{\mu}^{\mathrm{ess}}(V) .
$$

We consider a variant of the essential minimum. Let $j \in\{1, \ldots, n\}$ and $\theta \geq 0$. We define $V_{j}(\theta)$ as the subset of $\boldsymbol{\alpha} \in V(\overline{\mathbb{Q}})$ such that $\alpha_{i}$ is a root of unity for $i \neq j$ and $h\left(\alpha_{j}\right) \leq \theta$. We set $\hat{\mu}_{j}^{\text {ess }}(V)$ as the infimum of the set of $\theta \geq 0$ such that $V_{j}(\theta)$ is Zariski dense in $V$. Let $j \in\{1, \ldots, n\}$ and assume that the partial degrees $D_{j}$ satisfy $D_{j}>0$. In [1], proposition 2.7 (i) we prove the inequality

$$
D_{j} \hat{\mu}_{j}^{\text {ess }}(V) \leq \hat{h}(V) .
$$

Although this is not needed in the proof of the lower bounds for $\hat{h}(V)$, this inequality is in fact an equality. See Appendix, theorem 4.1.

Let $V=\{f=0\}$ be a $K$-irreducible hypersurface. In the second part of proposition 2.7 of [1], we deduced a new proof of Zhang's upper bound $\hat{\mu}^{\text {ess }}(V) \leq$ $\hat{h}(V) / \operatorname{deg}(V)$ from the inequality (2.2). As Martin Sombra pointed out, there is a mistake in the proof of this implication. If the polynomial $f\left(x_{1} x_{n}, \ldots, x_{n-1} x_{n}, x_{n}\right)$ is not necessarily irreducible over $K$, we cannot apply the result of the part (i) of that proposition.

\subsection{The stabilizer.}

We recall the definition and some properties of the stabilizer of a subvariety $V \subseteq$ $\mathbb{G}_{\mathrm{m}}^{n}$. We define the stabilizer of $V$ as the group

$$
\operatorname{Stab}(V)=\left\{\boldsymbol{\alpha} \in \mathbb{G}_{\mathrm{m}}^{n}, \boldsymbol{\alpha} V=V\right\} .
$$

We also denote by $\operatorname{Stab}(V)^{0}$ the connected component of $\operatorname{Stab}(V)$ containing the identity. Let $l$ be an integer. We remark that $l$ acts on $\mathbb{G}_{\mathrm{m}}^{n}$ by $\boldsymbol{\alpha} \mapsto \boldsymbol{\alpha}^{l}$. Let $k$ be the codimension of $\operatorname{Stab}(V)$ and assume that $V$ is geometrically irreducible. Then for any prime $p$ such that

$$
p \nmid\left[\operatorname{Stab}(V): \operatorname{Stab}(V)^{0}\right]
$$

$\operatorname{ker}[p] V$ is an union of $p^{k}$ distinct translates of $V$. 


\subsection{Hilbert Functions.}

Let $I \subset \overline{\mathbb{Q}}[\mathbf{x}]$ be an ideal and $\boldsymbol{\nu}=\left(\nu_{1}, \ldots, \nu_{n}\right) \in \mathbb{N}^{n}$. We denote the multihomogeneous Geometric Hilbert Function by

$$
H_{\mathrm{g}}(I ; \boldsymbol{\nu})=\operatorname{dim}\left(\overline{\mathbb{Q}}[\mathbf{x}]_{\boldsymbol{\nu}} / I_{\boldsymbol{\nu}}\right) .
$$

In this formula $\overline{\mathbb{Q}}[\mathbf{x}]_{\nu}$ is the vector space of polynomials of partial degrees $D_{j} \leq \nu_{j}$ and $I_{\nu}$ is its vector subspace $I \cap \overline{\mathbb{Q}}[\mathbf{x}]_{\nu}$.

Let $V \subset \mathbb{G}_{\mathrm{m}}^{n} \subseteq\left(\mathbb{P}_{1}\right)^{n}$ be an equidimensional reduced cycle, i. e. $V=W_{1} \cup \cdots \cup$ $W_{l}$ where $W_{1}, \ldots, W_{l}$ are geometrically irreducible of the same dimension. Let $I \subset \overline{\mathbb{Q}}[\mathbf{x}]_{\nu}$ be the ideal defining $V$ and let $I^{(T)}$ be the $T$-symbolic power of $I$, i. e. the ideal of polynomials vanishing with multiplicity $\geq T$ on the Zariski set defined by $I$. By abuse of notation, we set $H_{\mathrm{g}}(V ; \boldsymbol{\nu})=H_{\mathrm{g}}(I ; \boldsymbol{\nu})$ and $H_{\mathrm{g}}(V, T ; \boldsymbol{\nu})=H_{\mathrm{g}}\left(I^{(T)} ; \boldsymbol{\nu}\right)$. For a hypersurface $V$ of multi-degrees $\left(D_{1}, \ldots, D_{n}\right)$ we have:

$$
H_{\mathrm{g}}(V, T ; \boldsymbol{\nu})=\left(\nu_{1}+1\right) \cdots\left(\nu_{n}+1\right)-\left(\nu_{1}-T D_{1}+1\right) \cdots\left(\nu_{n}-T D_{n}+1\right) .
$$

We further need an Arithmetic Hilbert Function. Given a linear subspace $E \subseteq \overline{\mathbb{Q}}^{N}$ of dimension $L$, we define, following Schmidt ([11], Ch. 1, §. 8), its height as

$$
h_{L_{2}}(E)=\sum_{v} \frac{\left[K_{v}: \mathbb{Q}_{v}\right]}{[K: \mathbb{Q}]} \log \left\|\mathbf{w}_{1} \wedge \cdots \wedge \mathbf{w}_{N}\right\|_{v}
$$

where $\mathbf{w}_{1}, \ldots, \mathbf{w}_{N}$ is any basis of $E, K$ is a number field on which this basis is defined, $\|\cdot\|_{v}$ is the sup norm if $v \nmid \infty$ and

$$
\|\mathbf{w}\|_{v}^{2}=\sum_{j}\left|w_{j}\right|_{v}^{2}
$$

otherwise. Take $\boldsymbol{\nu}$ as before and set $N=\left(\nu_{1}+1\right) \cdots\left(\nu_{n}+1\right)$. We identify $\overline{\mathbb{Q}}[\mathbf{x}]_{\nu}$ with $\overline{\mathbb{Q}}^{N}$ by $\sum_{\boldsymbol{\lambda}} q_{\boldsymbol{\lambda}} \mathbf{x}^{\boldsymbol{\lambda}} \mapsto\left(q_{\boldsymbol{\lambda}}\right)_{0 \leq \lambda_{j} \leq \nu_{j}}$. Given a polynomial $F \in K[\mathbf{x}]_{\boldsymbol{\nu}}$ we set

$$
h_{L_{2}}(F)=\sum_{v} \frac{\left[k_{v}: \mathbb{Q}_{v}\right]}{[k: \mathbb{Q}]} \log \|F\|_{v} .
$$

Thus $h_{L_{2}}(F)=h_{L_{2}}(E)$ where $E \subseteq \overline{\mathbb{Q}}^{N}$ is the linear subspace generated by $F$. We recall Landau's theorem: if $P \in \mathbb{C}[\mathbf{x}]$ then $M(P)$ is bounded by the quadratic mean of its coefficients. Thus, if $F \in \mathbb{Z}[\mathbf{x}]$ vanishes on a reduced hypersurface $V$,

$$
\hat{h}(\{F=0\}) \leq h_{L_{2}}(F) .
$$

Let $V$ be an equidimensional reduced cycle as in the beginning of this subsection. We define its Arithmetic Hilbert Function as

$$
H_{\mathrm{a}}(V ; \boldsymbol{\nu})=h_{L_{2}}\left([I]_{\boldsymbol{\nu}}\right) .
$$




\section{Proof of the main results.}

The following lemma is the key ingredient for the proof of the main results.

Lemma 3.1 Let $\nu_{1}, \ldots, \nu_{n}, T$ be positive integers, $\left\{\boldsymbol{\alpha}_{1}, \ldots, \boldsymbol{\alpha}_{L}\right\} \subseteq \mathbb{P}_{n}(\mathbb{C})$, and $\boldsymbol{\lambda}_{1}, \ldots, \boldsymbol{\lambda}_{L}$ be multi-indexes such that $0 \leq \lambda_{i, j} \leq \nu_{j}$ for $i=1, \ldots, L$ and $j=$ $1, \ldots, n$. Define

$$
T_{0}:=\left(L-H_{\mathrm{g}}\left(\left\{\boldsymbol{\alpha}_{1}, \ldots, \boldsymbol{\alpha}_{L}\right\}, T ; \boldsymbol{\nu}\right)\right) T .
$$

Then the multi-homogeneous polynomial

$$
F\left(\mathbf{x}_{1}, \ldots, \mathbf{x}_{L}\right)=\operatorname{det}\left(\mathbf{x}_{i}^{\boldsymbol{\lambda}_{j}}\right)_{1 \leq i, j \leq L} .
$$

vanishes on $\left(\boldsymbol{\alpha}_{1}, \ldots, \boldsymbol{\alpha}_{L}\right) \in \mathbb{P}_{n}(\mathbb{C})^{L}$ with multiplicity at least $T_{0}$.

Proof. Let $S_{0}=\left\{\boldsymbol{\alpha}_{1}, \ldots, \boldsymbol{\alpha}_{L}\right\}$. If $H_{\mathrm{g}}\left(S_{0}, T ; \boldsymbol{\nu}\right) \geq L$ the assertion is obvious. Assume $H_{\mathrm{g}}\left(S_{0}, T ; \boldsymbol{\nu}\right)<L$ and let $L_{0}=L-H_{\mathrm{g}}\left(S_{0}, T ; \boldsymbol{\nu}\right)$. Then there exist linearly independent polynomials $G_{k}=\sum_{j=1}^{L} g_{k j} \mathbf{x}^{\boldsymbol{\lambda}_{j}}\left(k=1, \ldots, L_{0}\right)$ vanishing on $S_{0}$ with multiplicity $\geq T$. By elementary operations we replace the last $L_{0}$ columns of the matrix $\left(\mathbf{x}_{i}^{\boldsymbol{\lambda}_{j}}\right)$ by

$$
\left(G_{k}\left(\mathbf{x}_{1}\right), \ldots, G_{k}\left(\mathbf{x}_{L}\right)\right)^{t}, \quad k=1, \ldots, L_{0}
$$

(the exponent $t$ means "transpose"). Let $F^{\prime}\left(\mathbf{x}_{1}, \ldots, \mathbf{x}_{L}\right)$ be the determinant of this new matrix; then $F^{\prime}\left(\mathbf{x}_{1}, \ldots, \mathbf{x}_{L}\right)=c F\left(\mathbf{x}_{1}, \ldots, \mathbf{x}_{L}\right)$ for some $c \in \mathbb{C}^{*}$. The polynomials $G_{k}$ vanish on $S$ with multiplicity $\geq T$. Expanding $F^{\prime}\left(\mathbf{x}_{1}, \ldots, \mathbf{x}_{L}\right)$ with respect to the last $L_{0}$ columns we see that $F^{\prime}\left(\mathbf{x}_{1}, \ldots, \mathbf{x}_{L}\right)$ vanishes on $\left(\boldsymbol{\alpha}_{1}, \ldots, \boldsymbol{\alpha}_{L}\right) \in$ $\mathbb{P}_{n}(\mathbb{C})^{L}$ with the prescribed multiplicity.

In what follows we let $V \subseteq \mathbb{G}_{\mathrm{m}}^{n}$ be a geometrically irreducible hypersurface of multi-degrees $\left(D_{1}, \ldots, D_{n}\right)$.

Proposition 3.2 Let $\nu_{1}, \ldots, \nu_{n}, T$ be positive integers and let $p$ be a prime number. Let $h_{1}, \ldots, h_{n}$ be positive real numbers. Let $S$ be a subset of $\mathbb{G}_{\mathrm{m}}^{n}$ of points $\boldsymbol{\alpha}$ satisfying $h\left(\alpha_{i}\right) \leq h_{i}$ for $i=1, \ldots, n$. We assume that $S$ is Zariski dense in $V$. Then

$$
\begin{aligned}
\frac{H_{\mathrm{a}}(\operatorname{ker}[p] V ; \boldsymbol{\nu})}{H_{\mathrm{g}}(\operatorname{ker}[p] V ; \boldsymbol{\nu})} \leq-\left(1-\frac{H_{\mathrm{g}}(V, T ; \boldsymbol{\nu})}{H_{\mathrm{g}}(\operatorname{ker}[p] V ; \boldsymbol{\nu})}\right) \frac{T \log p}{p-1} & \\
& +\frac{n}{2} \log \left(\nu_{\max }+1\right)+\nu_{1} h_{1}+\cdots+\nu_{n} h_{n} .
\end{aligned}
$$

where $\nu_{\max }=\max \left\{\nu_{1}, \ldots, \nu_{n}\right\}$. 
Proof. For simplicity let $S^{\prime}=\operatorname{ker}[p] S, V^{\prime}=\operatorname{ker}[p] V$ and $N=\left(\nu_{1}+1\right) \cdots\left(\nu_{n}+1\right)$. We associate to $\boldsymbol{\beta} \in S^{\prime}$ the vector

$$
w_{\boldsymbol{\beta}}=\left(\boldsymbol{\beta}^{\boldsymbol{\lambda}}\right)_{0 \leq \lambda_{j} \leq \nu_{j}} \in \overline{\mathbb{Q}}^{N} .
$$

Let $E$ be the vector space generated by the $w_{\boldsymbol{\beta}}$ with $\boldsymbol{\beta} \in S^{\prime}$. Since $S$ is Zariski dense, $E^{\perp}=[I]_{\nu}$ where $I$ is the ideal defining $V^{\prime}$. We recall that $h_{L_{2}}(E)=$ $h_{L_{2}}\left(E^{\perp}\right)$ (see [11], Ch. 1, §8). Thus

$$
H_{\mathrm{a}}\left(V^{\prime} ; \boldsymbol{\nu}\right)=h_{L_{2}}(E) .
$$

Further

$$
L:=\operatorname{dim} E=H_{\mathrm{g}}\left(V^{\prime} ; \boldsymbol{\nu}\right) .
$$

We choose a basis $\boldsymbol{\beta}_{1}, \ldots, \boldsymbol{\beta}_{L}$ of $E$ and we denote for brevity $w_{j}=w_{\boldsymbol{\beta}_{j}}$. Let $v$ be a place. Then (see [11], proof of lemma $8 \mathrm{~A}$ ),

$$
\left\|\mathbf{w}_{1} \wedge \cdots \wedge \mathbf{w}_{L}\right\|_{v} \leq \prod_{j=1}^{L}\left\|\mathbf{w}_{j}\right\|_{v} .
$$

Moreover, for any $\boldsymbol{\beta} \in S^{\prime}$,

$$
\log \left\|\mathbf{w}_{\boldsymbol{\beta}}\right\|_{v} \leq \begin{cases}\prod_{i=1}^{n} \max \left\{1,\left|\beta_{i}\right|_{v}\right\}^{\nu_{j}}, & \text { if } v \nmid \infty ; \\ N^{1 / 2} \prod_{i=1}^{n} \max \left\{1,\left|\beta_{i}\right|_{v}\right\}^{\nu_{j}}, & \text { if } v \mid \infty .\end{cases}
$$

Thus, using the inequality $N \leq\left(\nu_{\max }+1\right)^{n}$,

$$
\begin{aligned}
& \log \left\|\mathbf{w}_{1} \wedge \cdots \wedge \mathbf{w}_{L}\right\|_{v} \\
& \quad \leq \begin{cases}\sum_{j=1}^{L} \sum_{i=1}^{n} \nu_{j} \log \max \left\{1,\left|\beta_{j, i}\right|_{v}\right\}, & \text { if } v \nmid \infty ; \\
\frac{n}{2} L \log \left(\nu_{\max }+1\right)+\sum_{j=1}^{L} \sum_{i=1}^{n} \nu_{j} \log \max \left\{1,\left|\beta_{j, i}\right|_{v}\right\}, & \text { if } v \mid \infty .\end{cases}
\end{aligned}
$$

We give a better bound for $v \mid p$. Let's choose distinct $\boldsymbol{\lambda}^{(1)}, \ldots, \boldsymbol{\lambda}^{(L)}$ with $0 \leq$ $\lambda_{j, i} \leq \nu_{j}$ for $j=1 \ldots, L$ and $i=1, \ldots, n$. We consider the determinant

$$
\Delta=\operatorname{det}\left(\boldsymbol{\beta}_{r}^{\boldsymbol{\lambda}_{s}}\right)_{r, s=1, \ldots, L}
$$

Let $\boldsymbol{\alpha}_{1}, \ldots, \boldsymbol{\alpha}_{L} \in S$ such that $\boldsymbol{\beta}_{j} \in \operatorname{ker}[p] \boldsymbol{\alpha}_{j}$ and set

$$
F\left(\mathbf{x}_{1}, \ldots, \mathbf{x}_{L}\right)=\operatorname{det}\left(\mathbf{x}_{r}^{\boldsymbol{\lambda}_{s}}\right)_{r, s=1, \ldots, L} .
$$

Thus $\Delta=F\left(\boldsymbol{\beta}_{1}, \ldots, \boldsymbol{\beta}_{L}\right)$ and, by lemma $(3.1), F$ vanishes on $\left(\boldsymbol{\alpha}_{1}, \ldots, \boldsymbol{\alpha}_{L}\right)$ with multiplicity at least

$$
T_{0}:=\left(L-H_{\mathrm{g}}\left(\left\{\boldsymbol{\alpha}_{1}, \ldots, \boldsymbol{\alpha}\right\}, T ; \boldsymbol{\nu}\right)\right) T \geq\left(L-H_{\mathrm{g}}(V, T ; \boldsymbol{\nu})\right) T .
$$


Since $v \mid p$, we have

$$
\left|\boldsymbol{\alpha}_{j, i}-\boldsymbol{\beta}_{j, i}\right|_{v} \leq p^{-1 /(p-1)}
$$

for $j=1, \ldots, L$ and $i=1, \ldots, n$. Thus, by Taylor expansion of $F$ around $\left(\boldsymbol{\alpha}_{1}, \ldots, \boldsymbol{\alpha}_{L}\right)$

$$
|\Delta|_{v}=\left|F\left(\beta_{1}, \ldots, \beta_{L}\right)\right|_{v} \leq p^{-T_{0} /(p-1)} \prod_{j=1}^{L} \prod_{i=1}^{n} \max \left\{1,\left|\beta_{j, i}\right|_{v}\right\}^{\nu_{j} L} .
$$

This formulas holds for the determinant of any $L \times L$ submatrix of the $L \times N$ matrix

$$
\left(\boldsymbol{\beta}_{j}^{\boldsymbol{\lambda}}\right)_{\substack{j=1, \ldots, L \\ 0 \leq \lambda_{i} \leq \nu_{i}}} .
$$

Thus,

$$
\log \left\|\mathbf{w}_{1} \wedge \cdots \wedge \mathbf{w}_{L}\right\|_{v} \leq-\frac{T_{0} \log p}{p-1}+\sum_{j=1}^{L} \sum_{i=1}^{n} \nu_{j} \log \max \left\{1,\left|\beta_{j, i}\right|_{v}\right\} .
$$

By (3.6), (3.7) and (3.8) we obtain:

$$
H_{\mathrm{a}}\left(V^{\prime} ; \boldsymbol{\nu}\right) \leq-\frac{T_{0} \log p}{p-1}+\frac{n}{2} L \log \left(\nu_{\max }+1\right)+\left(\nu_{1} h_{1}+\cdots+\nu_{n} h_{n}\right) L .
$$

Proposition 3.2 follows.

Choosing the parameters in a suitable way, we deduce

Proposition 3.3 For any prime number $p$,

$$
\hat{h}(V) \geq \frac{\log p}{7 p}-\frac{n k \log p}{p^{k}}-\frac{n \log \left(n^{2} D_{\max }\right)}{2 p^{k}},
$$

where $k$ is the codimension of the stabilizer of $V$ and where $D_{\max }=\max \left\{D_{1}, \ldots, D_{n}\right\}$

Proof. Let us assume first that $p \nmid\left[\operatorname{Stab}(V): \operatorname{Stab}(V)^{0}\right]$, so that (see subsection 2.2) $V^{\prime}=\operatorname{ker}[p] V$ is a union of $p^{k}$ translates of $V$. We show in this case that

$$
\hat{h}(V) \geq \frac{\log p}{7 p}-\frac{n k \log p}{p^{k}}-\frac{n \log \left(n D_{\max }\right)}{2 p^{k}} .
$$

Let $\varepsilon>0$. Assume $D_{\max }=D_{n}$. By proposition 2.7 (i) of [1] the set $S=\left\{\left(\zeta_{1}, \ldots, \zeta_{n-1}, \alpha\right) \in V(\overline{\mathbb{Q}}), \zeta_{1}, \ldots, \zeta_{n-1}\right.$ roots of unity, $\left.h(\alpha) \leq \hat{h}(V) / D_{n}+\varepsilon\right\}$ 
is Zariski dense in $V$. We apply proposition 3.2 with $h_{1}=\cdots=h_{n-1}=0$ and $h_{n}=\hat{h}(V) / D_{n}+\varepsilon$. We choose

$$
\left\{\begin{array}{l}
\nu_{j}=n p^{k} D_{j}-1, \quad \text { for } j=1, \ldots, n-1 \\
\nu_{n}=p^{k} D_{n}-1
\end{array}\right.
$$

and

$$
T=\left[p^{k} / 2\right] \text {. }
$$

We remark that $\nu_{\max }=\max \left\{\nu_{1}, \ldots, \nu_{n}\right\} \leq n p^{k} D_{\max }-1$. Further, by (2.3)

$$
\begin{aligned}
H_{\mathrm{g}}(V, T ; \boldsymbol{\nu}) & =\left(\nu_{1}+1\right) \cdots\left(\nu_{n}+1\right)-\left(\nu_{1}-T D_{1}+1\right) \cdots\left(\nu_{n}-T D_{n}+1\right) \\
& \leq n^{n-1} p^{k n} D_{1} \cdots D_{n}-\frac{1}{2}\left(n-\frac{1}{2}\right)^{n-1} p^{k n} D_{1} \cdots D_{n}
\end{aligned}
$$

and

$$
\begin{aligned}
H_{\mathrm{g}}\left(V^{\prime} ; \boldsymbol{\nu}\right) & =\left(\nu_{1}+1\right) \cdots\left(\nu_{n}+1\right)-\left(\nu_{1}-p^{k} D_{1}+1\right) \cdots\left(\nu_{n}-p^{k} D_{n}+1\right) \\
& =n^{n-1} p^{k n} D_{1} \cdots D_{n}
\end{aligned}
$$

so that

$$
1-\frac{H_{\mathrm{g}}(V, T ; \boldsymbol{\nu})}{H_{\mathrm{g}}\left(V^{\prime} ; \boldsymbol{\nu}\right)} \geq \frac{1}{2}\left(1-\frac{1}{2 n}\right)^{n-1} \geq \frac{1}{2 \sqrt{e}} .
$$

Inequality (3.5) gives (forgetting the positive contribution of the Arithmetic Hilbert Function)

$$
\begin{aligned}
\nu_{n} h_{n} & \geq \frac{T \log p}{2 \sqrt{e} p}-\frac{n}{2} \log \left(\nu_{\max }+1\right) \\
& \geq \frac{p^{k} \log p}{4 \sqrt{e} p}-\frac{\log p}{2 \sqrt{e} p}-\frac{n}{2} \log \left(n p^{k} D_{n}\right) \\
& \geq \frac{p^{k} \log p}{7 p}-n k \log p-\frac{n}{2} \log \left(n D_{n}\right) .
\end{aligned}
$$

Further,

$$
\nu_{n} h_{n}=\left(p^{k} D_{n}-1\right)\left(\frac{\hat{h}(V)}{D_{n}}+\varepsilon\right) \leq p^{k}\left(\hat{h}(V)+\varepsilon D_{n}\right) .
$$

Thus

$$
\hat{h}(V)+\varepsilon D_{n} \geq \frac{\log p}{7 p}-\frac{n k \log p}{p^{k}}-\frac{n \log \left(n D_{\max }\right)}{2 p^{k}} .
$$

By letting $\varepsilon \rightarrow 0$ we obtain the lower bound (3.10). 
We now consider the general case, when $\operatorname{Stab}(V)$ is not necessarily connected. Proposition 2.4 of $[1]^{1}$ gives a hypersurface $W=\{H=0\}$ with connected stabilizer of the same codimension $k$ and normalized height $\hat{h}(W) \leq \hat{h}(V)$. Let $r$ be the rank of the finite abelian group $\operatorname{Stab}(V) / \operatorname{Stab}(V)^{0}$ and let $d_{r}|\cdots| d_{1}$ its elementary divisors. By inspection of the proof of this proposition, we see that (after eventually renumbering the coordinates)

$$
D_{j}^{\prime}:=\operatorname{deg}_{x_{j}}(H)= \begin{cases}\left(j-1+1 / d_{j}\right) D_{j} & \text { if } j=1, \ldots, r \\ (r+1) D_{j} & \text { if } j=r+1, \ldots, n .\end{cases}
$$

Thus $W$ has multi-degree $\left(D_{1}^{\prime}, \ldots, D_{n}^{\prime}\right)$ with $D_{j}^{\prime} \leq n D_{j}$. By inequality (3.10) we deduce

$$
\hat{h}(V) \geq \hat{h}(W) \geq \frac{\log p}{7 p}-\frac{n k \log p}{p^{k}}-\frac{n \log \left(n^{2} D_{\max }\right)}{2 p^{k}} .
$$

We now assume $k=n$, i. e. $\operatorname{Stab}(V)$ discrete. Choosing $p=5$ we obtain

Theorem 3.4 Assume that $\operatorname{Stab}(V)$ is discrete, $n \geq 9$ and

$$
\max D_{j} \leq 3^{2^{n}} .
$$

Then

$$
\hat{h}(V) \geq \frac{1}{23}
$$

Proof. We apply the above proposition with $p=5$ and $k=n$. By assumption $D_{\max } \leq 3^{2^{n}}$. We obtain

$$
\begin{aligned}
\hat{h}(V) & \geq \frac{\log 5}{35}-\frac{n^{2} \log 5}{5^{n}}-\frac{n \log \left(n^{2} D_{\max }\right)}{2 \times 5^{n}} \\
& \geq \frac{\log 5}{35}-\frac{n^{2} \log 5}{5^{n}}-\frac{2 n \log n}{2 \times 5^{n}}-\frac{n 2^{n} \log 3}{2 \times 5^{n}}=: f(n) .
\end{aligned}
$$

An easy computation shows that $f$ is an increasing function and $f(9)>1 / 23$.

\footnotetext{
${ }^{1}$ In this proposition, the authors assume that $V$ is defined over $\mathbb{Q}$ and $\mathbb{Q}$-irreducible. Nevertheless, the proof of the proposition can immediately be generalized to a geometrically irreducible hypersurface.
} 
We conclude this section with a more general and technical lower bound for the normalized height of a hypersurface.

Theorem 3.5 Assume that $V$ is not a translate of a torus. Then

$$
\hat{h}(V) \geq \frac{1}{56} \times \max \left(\frac{\log \left(n \log \left(n^{2} D_{\max }\right)\right)}{k}, 1\right) \times\left(\frac{\log \left(n \log \left(n^{2} D_{\max }\right)\right)}{28 n k \log \left(n^{2} D_{\max }\right)}\right)^{1 /(k-1)}
$$

where $k$ is the codimension of the stabilizer of $V$ and $D_{\max }=\max D_{j}$. In particular

$$
\hat{h}(V) \geq \frac{\log \left(n \log \left(n^{2} D_{\max }\right)\right)^{2}}{6272 n \log \left(n^{2} D_{\max }\right)} .
$$

Proof. Let

$$
N=\left(\frac{28 n k \log \left(n^{2} D_{\max }\right)}{\log \left(n \log \left(n^{2} D_{\max }\right)\right)}\right)^{1 /(k-1)} .
$$

Let us choose a prime $p$ such that $N \leq p \leq 2 N$. Since for any $x>0$

$$
\log x \leq x^{1 / 2}
$$

we have $\log \left(n \log \left(n^{2} D_{\max }\right)\right) \leq \log \left(n\left(n^{2} D_{\max }\right)^{1 / 2}\right) \leq \log \left(n^{2} D_{\max }\right)$. Hence

$$
p^{k-1} \geq N \geq 28 n k \text {. }
$$

Moreover relation (3.12) gives

$$
\log p \geq \log N \geq \frac{\log \left(28 n^{1 / 2} k \log \left(n^{2} D_{\max }\right)^{1 / 2}\right)}{k-1} \geq \frac{\log \left(n \log \left(n^{2} D_{\max }\right)\right)}{2 k} .
$$

Therefore

$$
p^{k-1} \log p \geq N^{k-1} \log p \geq 14 n \log \left(n^{2} D_{\max }\right) .
$$

By proposition 3.3 we deduce

$$
\begin{aligned}
\hat{h}(V) & \geq \frac{\log p}{7 p}-\frac{n k \log p}{p^{k}}-\frac{n \log \left(n^{2} D_{\max }\right)}{2 p^{k}} \\
& \geq \frac{\log p}{7 p}-\frac{\log p}{28 p}-\frac{\log p}{28 p} \\
& =\frac{\log p}{14 p} .
\end{aligned}
$$

By relation (3.13) and by the trivial bound $\log p \geq \log 2$ we obtain

$$
\begin{aligned}
\hat{h}(V) & \geq \frac{1}{14} \times \max \left(\frac{\log \left(n \log \left(n^{2} D_{\max }\right)\right)}{2 k}, \log 2\right) \times \frac{1}{2 N} \\
& \geq \frac{1}{56} \times \max \left(\frac{\log \left(n \log \left(n^{2} D_{\max }\right)\right)}{k}, 1\right) \times\left(\frac{\log \left(n \log \left(n^{2} D_{\max }\right)\right)}{28 n k \log \left(n^{2} D_{\max }\right)}\right)^{1 /(k-1)} .
\end{aligned}
$$


This proves the first inequality of theorem 3.5. For the second one, we remark that $k \geq 2$ and $k(n k)^{1 /(k-1)} \leq 4 n$. Thus

$$
\hat{h}(V) \geq \frac{\log \left(n \log \left(n^{2} D_{\max }\right)\right)^{2}}{56 \times 28 \times 4 n \log \left(n^{2} D_{\max }\right)}=\frac{\log \left(n \log \left(n^{2} D_{\max }\right)\right)^{2}}{6272 n \log \left(n^{2} D_{\max }\right)} .
$$

Remark 3.6 Let $K$ be a number field and let $V$ be any hypersurface defined and $i r$ reducible over $K$, of multidegree $\left(D_{1}, \ldots, D_{n}\right)$. Let $W$ be a geometrically irreducible component of $V$, of multidegree $\left(\delta_{1}, \ldots, \delta_{n}\right)$. Then $\operatorname{dim} \operatorname{Stab} W=\operatorname{dim} \operatorname{Stab} V$, $\delta_{j} \leq D_{j}$ and $\hat{h}(W) \leq \hat{h}(V)$. Thus, theorems 3.4 and 3.5 apply to a hypersurfaces defined and irreducible over a number field $K$. In particular, we deduce from theorem 3.4 the lower bound for the Mahler measure of a polynomial $f \in \mathbb{Z}\left[x_{1}, \ldots, x_{n}\right]$ announced in the introduction.

\section{Appendix. Normalized height and essential mini- mum}

Let $V \subseteq \mathbb{G}_{\mathrm{m}}^{n}$ be a hypersurface of multi-degrees $\left(D_{1}, \ldots, D_{n}\right)$ defined and irreducible over some number field $K$. We prove:

Theorem 4.1 Let $j \in\{1, \ldots, n\}$ and assume $D_{j}>0$. Then

$$
\hat{h}(V)=D_{j} \hat{\mu}_{j}^{\mathrm{ess}}(V) \text {. }
$$

Proof. We can assume $j=n$. We have already remarked that the inequality $D_{n} \hat{\mu}_{n}^{\text {ess }}(V) \leq \hat{h}(V)$ is proved in [1], proposition 2.7 (i). Hence, it is enough to prove

$$
\hat{h}(V) \leq D_{n} \hat{\mu}_{n}^{\text {ess }}(V) .
$$

Let $W_{1}, \ldots, W_{s}$ be the geometrically irreducible components of $V$. Then $\hat{\mu}_{n}^{\text {ess }}(V)=$ $\hat{\mu}_{n}^{\text {ess }}\left(W_{j}\right), \hat{h}(V)=s \hat{h}\left(W_{s}\right)$ and $D_{n}=s \operatorname{deg}_{x_{n}}\left(W_{j}\right)$. Thus we can assume that $V$ is geometrically irreducible. Let $\theta>\hat{\mu}_{n}^{\text {ess }}(V)$ and let $p$ be a prime number. Let also $k \geq 1$ be the codimension of the stabilizer of $V$. We apply proposition 3.2 with $h_{1}=\cdots=h_{n-1}=0, h_{n}=\theta$ and

$$
\nu_{j}= \begin{cases}\lambda p^{k} D_{j}-1, & \text { if } j=1, \ldots, n-1 \\ \mu p^{k} D_{n}-1, & \text { if } j=n\end{cases}
$$

where $\lambda$ and $\mu$ are positive integers. We further set

$$
T=\left[P^{k} / 2\right] \text {. }
$$


Inequality (3.5) gives (forgetting the extra contribution at the place dividing $p$ ):

$$
\frac{H_{\mathrm{a}}(\operatorname{ker}[p] V ; \boldsymbol{\nu})}{H_{\mathrm{g}}(\operatorname{ker}[p] V ; \boldsymbol{\nu})} \leq \frac{n}{2} \log \left(\nu_{\max }+1\right)+\nu_{n} \theta .
$$

Let $\varepsilon>0$. The Absolute Siegel's lemma of Zhang (see [5], lemme 4.7 and the remark which follows) shows that for any $\varepsilon>0$ there exists a non-zero point $\mathbf{x} \in S$ such that

$$
h_{L_{2}}(\mathbf{x}) \leq \frac{h_{L_{2}}(S)}{\operatorname{dim}(S)}+\frac{\log \operatorname{dim}(S)}{2}+\varepsilon .
$$

We choose in this statement $S=[I]_{\nu}$, with $I$ the ideal of definition of $\operatorname{ker}[p] V$. Let $N=\left(\nu_{1}+1\right) \cdots\left(\nu_{n}+1\right)$ and assume that $H_{\mathrm{g}}(\operatorname{ker}[p] V ; \boldsymbol{\nu})<N$. We have $h_{L_{2}}(S)=H_{\mathrm{a}}(\operatorname{ker}[p] V ; \boldsymbol{\nu})$ and

$$
\operatorname{dim}(S)=N-H_{\mathrm{g}}(\operatorname{ker}[p] V ; \boldsymbol{\nu})<N \leq\left(\nu_{\max }+1\right)^{n} .
$$

Let

$$
\varepsilon=\frac{n}{2} \log \left(\nu_{\max }+1\right)-\frac{\log \operatorname{dim}(S)}{2}>0 .
$$

By (4.14) and (4.15) there exists a non-zero $F \in \overline{\mathbb{Q}}[\mathbf{x}]_{\nu}$ vanishing on $\operatorname{ker}[p] V$ and such that

$$
\begin{aligned}
h_{L_{2}}(F) & \leq \frac{H_{\mathrm{a}}(\operatorname{ker}[p] V ; \boldsymbol{\nu})}{N-H_{\mathrm{g}}(\operatorname{ker}[p] V ; \boldsymbol{\nu})}+\frac{n}{2} \log \left(\nu_{\max }+1\right) \\
& \leq \frac{H_{\mathrm{g}}(\operatorname{ker}[p] V ; \boldsymbol{\nu})}{N-H_{\mathrm{g}}(\operatorname{ker}[p] V ; \boldsymbol{\nu})} \nu_{n} \theta+\frac{N}{N-H_{\mathrm{g}}(\operatorname{ker}[p] V ; \boldsymbol{\nu})} \frac{n}{2} \log \left(\nu_{\max }+1\right) .
\end{aligned}
$$

By Landau's theorem (see (2.4)) $p^{k} \hat{h}(V) \leq h_{L_{2}}(F)$. Thus

$$
\hat{h}(V) \leq \frac{H_{\mathrm{g}}(\operatorname{ker}[p] V ; \boldsymbol{\nu})}{N-H_{\mathrm{g}}(\operatorname{ker}[p] V ; \boldsymbol{\nu})} p^{-k} \nu_{n} \theta+\frac{N}{N-H_{\mathrm{g}}(\operatorname{ker}[p] V ; \boldsymbol{\nu})} \frac{n}{2 p^{k}} \log \left(\nu_{\max }+1\right) .
$$

We have

$$
N=\lambda^{n-1} \mu p^{n k} D_{1} \cdots D_{n}
$$

and, by $(2.3)$,

$$
N-H_{\mathrm{g}}(\operatorname{ker}[p] V ; \boldsymbol{\nu})=(\lambda-1)^{n-1}(\mu-1) p^{n k} D_{1} \cdots D_{n} .
$$

Thus

$$
\begin{aligned}
\hat{h}(V) \leq \frac{\lambda^{n-1} \mu-(\lambda-1)^{n-1}(\mu-1)}{(\lambda-1)^{n-1}(\mu-1)} \mu D_{n} \theta & \\
& \quad+\frac{\lambda^{n-1} \mu}{(\lambda-1)^{n-1}(\mu-1)} \frac{n}{2 p^{k}}(\log (\max \{\lambda, \mu\})+k \log p) .
\end{aligned}
$$


Taking $p \rightarrow+\infty$ and $\theta \rightarrow \hat{\mu}_{n}^{\text {ess }}(V)$ we obtain:

$$
\hat{h}(V) \leq \frac{\lambda^{n-1} \mu-(\lambda-1)^{n-1}(\mu-1)}{(\lambda-1)^{n-1}(\mu-1)} \mu D_{n} \hat{\mu}_{n}^{\text {ess }}(V) .
$$

It is now enough to remark that

$$
\lim _{\mu \rightarrow+\infty} \lim _{\lambda \rightarrow+\infty} \frac{\lambda^{n-1} \mu-(\lambda-1)^{n-1}(\mu-1)}{(\lambda-1)^{n-1}(\mu-1)} \mu=\lim _{\mu \rightarrow+\infty} \frac{\mu}{\mu-1}=1 .
$$

\section{References}

[1] F. Amoroso and S. David. "Minoration de la hauteur normalisée des hypersurfaces". Acta Arith. 92, no. 4 (2000), 340-366.

[2] F. Amoroso et S. David. "Minoration de la hauteur normalisée dans un tore", Journal de l'Institut de Mathématiques de Jussieu, 2, no. 3 (2003), $335-381$.

[3] D. Boyd. "Kronecker's theorem and Lehmer's problem for polynomials in several variables". J. of Number Theory, 13 (1980), 116-121.

[4] D. Boyd, "Speculations concerning the range of Mahler's measure", Can. Math. Bull. 24 (1981), 453-469.

[5] S. David and P. Philippon. "Minorations des hauteurs normalisées des sous-variétés des tores". Ann. Scuola Norm. Sup. Pisa Cl. Sci. (4), 28 (1999), no. 3, 489-543; Errata, ibidem, 29, no 3 (2000), 729-731.

[6] E. Dobrowolski. "On a question of Lehmer and the number of irreducible factors of a polynomial". Acta Arith., 34 (1979), 391-401.

[7] W. Lawton. "A generalization of a theorem of Kronecker". Journal of the Science Faculty of the Chiangmai University (Thaillande), 4 (1977), $15-23$.

[8] W. Lawton. "A problem of Boyd concerning geometric means of polynomials". J. of Number Theory, 16 (1983), 356-362.

[9] D. H. Lehmer. "Factorization of certain cyclotomic functions". Ann. of Math. 34 (1933), 461-479.

[10] G. Myerson and C. J. Smyth, "On measures of polynomials in several variables; corrigendum". Bull. Austral. Math. Soc. 26 (1982), 317-319. 
[11] W. M. Schmidt. Diophantine approximation and Diophantine equations. Springer Lecture Notes in Mathematics, t. 1467, Springer-Verlag, Berlin-Heidelberg-New-York, viii + 217 pages, 1991.

[12] C. J. Smyth. "On measures of polynomials in several variables". Bull. Austral. Math. Soc. 23 (1981), 49-63.

[13] C. J. Smyth, "A Kronecker-type theorem for complex polynomials in several variables". Canad. Math. Bull., 24 (1981), 447-452. Errata, ibidem, 25 (1982), 504.

[14] P. Voutier. "An effective lower bound for the height of algebraic numbers". Acta Arith., 74 (1996), 81-95.

[15] S. Zhang. "Positive line bundles on arithmetic varieties". J. Amer. Math. Soc., 8, no. 1 (1995), 187-221.

Laboratoire de mathématiques Nicolas Oresme, CNRS UMR 6139

Université de Caen, Campus II, BP 5186

14032 Caen Cédex, France 